\title{
Coinfección Leishmania infantum, VIH e Histoplasma capsulatum: manifestaciones dermatológicas
}

\author{
Lilian Chena ${ }^{\mathrm{I}, \mathrm{V}}$, Eva Nara ${ }^{\mathrm{I}}$, Andrés Canese ${ }^{\mathrm{II}}$,Gustavo Aguilar ${ }^{\mathrm{III}}$, Patricia Araujo ${ }^{\mathrm{III}}$, Aurelia Taboada ${ }^{\mathrm{IV}}$, \\ Myriam Moránv, Graciela Russomando ${ }^{\mathrm{I}}$ \\ I Departamento de Biología Molecular Biotecnología, IICS, UNA. Paraguay \\ II Programa Nacional de Control de Leishmaniosis, SENEPA, MSP y BS. Paraguay \\ III Departamento de Micología, LCSP, MSP y BS. Paraguay \\ IV Instituto de Medicina Tropical, MPS y BS. Paraguay \\ V Departamento de Parasitología, LCSP, MSP y BS. Paraguay
}

Cómo referenciar este artículo/ How to reference this article:
Chena L, Nara E, Canese A, Aguilar G, Araujo P, Taboada A et al. Coinfección Leishmania infantum, VIH e Histoplasma capsulatum: manifestaciones dermatológicas. Mem. Inst. Investig. Cienc. Salud. 2016;14(3):121-125

\section{R E S U M E N}

La coinfección Leishmania/VIH es frecuente y por lo general son casos de leishmaniosis visceral (LV), sin embargo, Leishmania infantum, el agente etiológico, también puede causar formas cutáneas en pacientes VIH-positivos. Los parásitos llegan a la piel por difusión, en una nueva infección o reactivación de una infección latente. Existen técnicas moleculares que confirman el diagnóstico y caracterizan a la especie. En estos pacientes también se presenta como infección oportunista la histoplasmosis, reconocida como marcador de SIDA y causada por Histoplasma capsulatum. En este trabajo describimos un caso de coinfección SIDA, histoplasmosis y LV asociado a lesiones cutáneas. Paciente de sexo masculino, adulto, residente en J.A.Saldívar, VIH positivo (2010), sin tratamiento antirretroviral. En el 2012 es internado en el Instituto de Medicina Tropical (IMT) con fiebre, anemia, hepatoesplenomegalia, frotis de médula ósea y rk39 negativos para Leishmania, recibe tratamiento con Anfotericina B. En el 2013 presenta síndrome febril prolongado y en médula ósea se encuentran amastigotes de Leishmania sp. En el 2014 presenta úlceras en el rostro en las que se observan es porosmicóticos de $H$. capsulatum y amastigotes de Leishmania $s p$. Se confirma L. infantum por técnicas de biología molecular. En el caso estudiado concluimos que la presentación dérmica es causada por $L$. infantum, caracterizada por técnicas moleculares, eH. capsulatum que se presenta en concomitancia debido al estado inmunológico. Cabe resaltar la importancia del diagnóstico diferencial para aplicar el tratamiento correcto y además la manifestación clínica que normalmente no es asociada a esta especie de Leishmania.

Palabras claves: Leishmania infantum, Histoplasma capsulatum, VIH, lesiones cutáneas

\section{Leishmania infantum, HIV and Hystoplasma capsulatum coinfection: dermatologic manifestations}

\section{A B S T R A C T}

Leishmania/HIV coinfection is frequent and generally they are visceral leishmaniasis cases $(\mathrm{VL})$. However, Leishmania infantum, the etiological agent, could also cause cutaneous forms in $H I V$-positive patients. The parasites reach the skin through diffusion, a new infection or a reactivation of a latent infection. There are molecular techniques that confirm the diagnosis and characterize Leishmania species. These patients also present other opportunistic infections like histoplasmosis, well known as an AIDS marker and caused by Histoplasma capsulatum. In this work, we describe one HIV/histoplasmosis/VL coinfection case associated to cutaneous lesions. The patient was a male, adult, coming from the city of J.A. Saldívar, HIV positive (since 2010), without antiretroviral treatment. He was 
hospitalized in 2012 at the Instituto de Medicina Tropical (IMT) with fever, anemia, hepatosplenomegaly, bone marrow smear and rK39 both negative for Leishmania. He was treated with Amphotericin B. In 2013, he presented a prolonged febrile syndrome and Leishmania amastigotes were observed in the bone marrow. In 2014, the patient presented ulcerations on the face where $H$. capsulatum fungal spores and Leishmania amastigotes were observed. $L$. infantum was confirmed by molecular methods. In the case under study we can conclude that the dermal manifestation was caused by $L$. infantuma ccording to the characterization by molecular techniques, and simultaneously $H$. capsulatum was present due to his immunological status. It should be emphasized the importance of the differential diagnosis in order to apply the right treatment and also the uncommon clinical manifestation that is not associated to this Leishmania species.

Keywords: Leishmania infantum, Histoplasma capsulatum, HIV, cutaneous lesions

\section{INTRODUCCIÓN}

Las manifestaciones cutáneas del síndrome de inmunodeficiencia adquirida (SIDA) han sido ampliamente reconocidas desde la aparición de esta enfermedad ${ }^{(1,2)}$. Las alteraciones que se observan en la mayoría de los casos son el resultado del estado inmunológico deficiente del paciente y suelen ser la primera expresión de la enfermedad y un marcador progresivo. Las de origen infeccioso son frecuentes y los microorganismos causales son los que en la población inmunocompetente de manera habitual producen síntomas leves o concurren sin síntomas manifiestos ${ }^{(1)}$. La inmunosupresión inherente al SIDA permite la aparición de enfermedades oportunistas con cuadros clínicos diferentes, de diagnóstico y tratamiento difíciles ${ }^{(3)}$.

La coinfección Leishmania/VIH es observada con frecuencia en los últimos años en países de América Latina y por lo general son casos de Leishmaniosis Visceral (LV) cuyo agente etiológico es Leishmania infantum, que pertenece al complejo Leishmania donovani ${ }^{(4,5)}$. Sin embargo, esta especie también puede causar formas cutáneas en pacientes VIH positivos. Los parásitos llegan a la piel por difusión, en una nueva infección o reactivación de una infección latente ${ }^{(5,6)}$. Los pacientes coinfectados clásicamente presentan un curso clínico crónico, con altas tasas de fracaso del tratamiento y recaídas ${ }^{(7)}$.

Para el diagnóstico, además de las técnicas convencionales como la observación microscópica, cultivo de muestras y anatomía patológica, existen técnicas moleculares que confirman y permiten identificar el género y caracterizar los complejos de Leishmania ${ }^{(8,9)}$.

En estos pacientes también se presenta como infección oportunista la histoplasmosis, reconocida como marcador de SIDA y es causada por Histoplasma capsulatum ${ }^{(10,11)}$. Es considerada clásicamente una micosis endémica en América Latina y otros países tropicales ${ }^{(12,13)}$, sin embargo el factor de riesgo más importante para la reactivación de la infección y progresión de la enfermedad es la inmunosupresión inducida por el $V_{I H}{ }^{(14,15)}$. La histoplasmosis puede manifestarse como reactivación de la infección primaria, producto del contacto previo con el agente etiológico o como infección reciente (10). En sujetos inmunodeprimidos, sobre todo en pacientes con SIDA cuando el recuento de CD4+ es menor a 50 células $/ \mathrm{mL}$, la histoplasmosis puede presentarse en forma de una infección grave y diseminada ${ }^{(16)}$. En estos pacientes la infección puede cursar con lesiones cutáneas, ocurre en un $4-11 \%$ de los casos como resultado de la invasión secundaria de la piel por la diseminación hematógena del hongo ${ }^{(17)}$ Las lesiones son polimorfas se inician como máculas o pápulas, y posteriormente aumentan en número y tamaño, originando nódulos y a veces úlceras, localizados en rostro o generalizados ${ }^{(12,16,18)}$.

En este trabajo describimos un caso de coinfección SIDA, histoplasmosis y LV asociado a lesiones cutáneas, el diagnóstico diferencial y la caracterización molecular de L. infantum.

\section{PRESENTACIÓN DEL CASO}

Paciente de sexo masculino, adulto, residente en el distrito de J. A. Saldívar, Departamento Central, VIH positivo diagnosticado en el 2010, al momento del diagnóstico, recuento de $\mathrm{CD} 4+180$ células $/ \mathrm{mL}$. Inicia tratamiento antirretroviral con Zidovudina, Lamivudina y Nevirapina, aunque poco adherente a sus consultas y medicación. Posteriormente se registran varios abandonos de medicación.

En el 2012 ingresa al Instituto de Medicina Tropical (IMT) con un cuadro de fiebre, anemia, anorexia, pérdida de peso, hepato-esplenomegalia, además sinusitis y herpes labial. Se realiza frotis de médula ósea y la prueba serológica rk 39, ambos son negativos para Leishmania, los datos de laboratorio registran glóbulos blancos $1.820 / \mathrm{mm} 3$, 
hematocrito $30 \%$ y recuento de plaquetas $90.000 / \mathrm{mm} 3$. Recibe tratamiento con Anfotericina B.

En el 2013 presenta síndrome febril prolongado y en frotis de médula ósea se encuentran abundantes formas amastigotes de Leishmania $s p$, además se diagnostica tuberculosis diseminada y candidiasis oral. Recuento de CD4+: 1 célula/mL. Paciente desaparece del servicio.

En el 2014 nuevamente ingresa al IMT, presenta lesión en cara y miembro superior izquierdo, al inicio costrosa que luego se hace ulcerosa. Consulta por fiebre de un mes, tos con expectoración de dos semanas de evolución y lesiones ulcerosas de las que no sabe definir tiempo de evolución. Recuento de CD4+ 6 células $/ \mathrm{mL}$. Se realiza frotis de la lesión cutánea y se observan esporos micóticos en semiluna de Histoplasma capsulatum y formas amastigotes de Leishmania sp, visualizados con tinción de Giemsa (Figura 1). La muestra es remitida para confirmación y caracterización molecular. Por la técnica de PCR se identifica ADN del género Leishmania (120 pb) y del complejo L. donovani (100 pb) y se confirma como L. infantum (Figura 2).

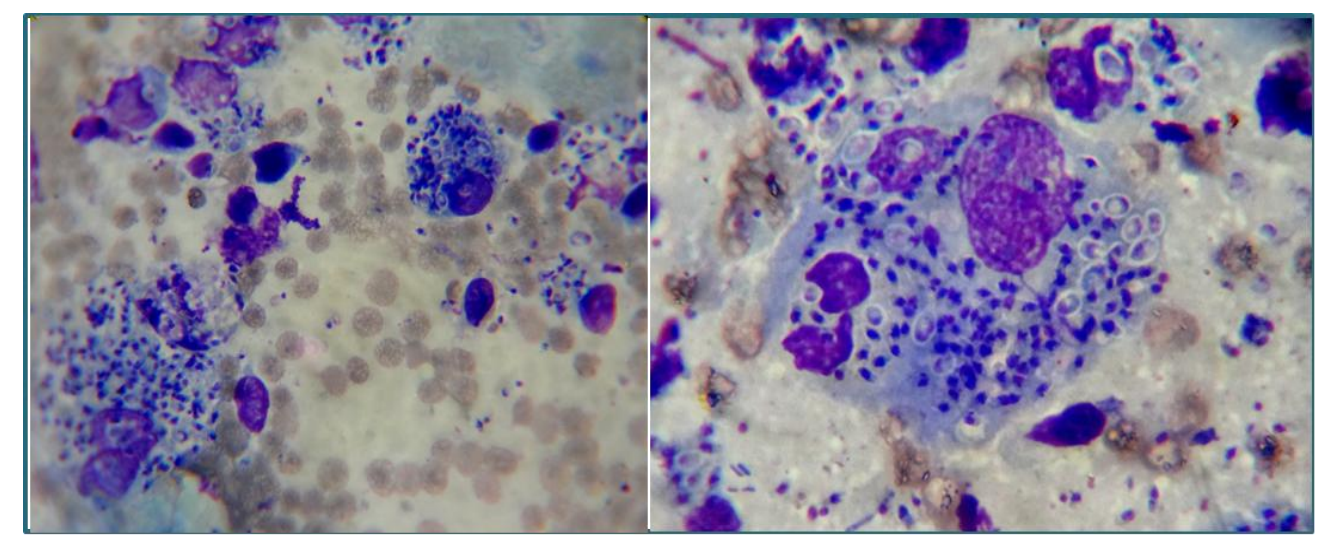

Figura 1. Esporos micóticos en semiluna de Histoplasma capsulatum y amastigotes de Leishmania en frotis de lesión cutánea.

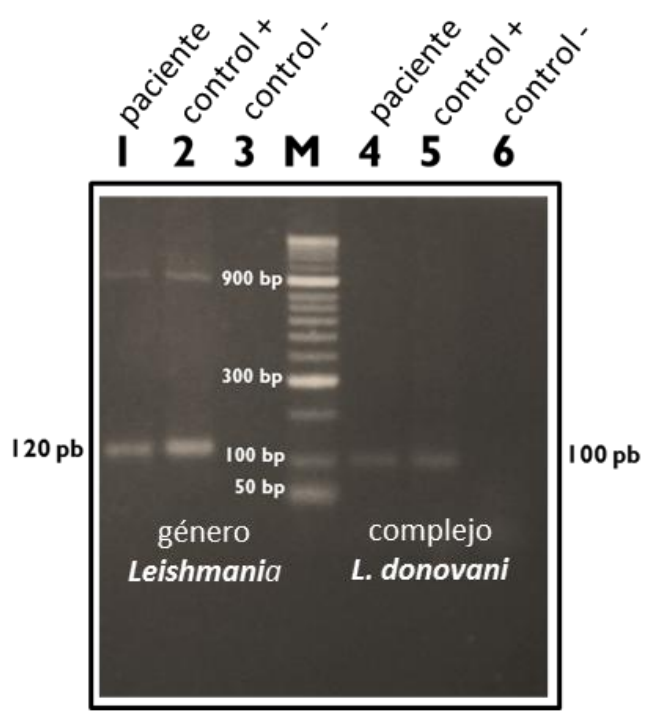

Figura 2. Producto de amplificación de kDNA de Leishmania (120 pb) y de secuencias repetitivas de $L$. infantum (100 pb). Carriles 1 y 4: pacientes, Carril 2: control positivo (género Leishmania), Carril 5: control positivo (complejo L. donovani), Carriles 3 y 6: control negativo, Carril M, marcador de peso molecular de 100 pb (BIOLINE, UK) 
Presenta diagnóstico de tuberculosis pulmonar probable, leishmaniosis cutánea, histoplasmosis cutánea, anemia severa, insuficiencia respiratoria aguda, shock séptico pulmonar a Klebsiella pneumoniae. Se registra su deceso en setiembre del 2014.

\section{DISCUSIÓN}

Debido a las infecciones oportunistas que pueden ocurrir en pacientes con VIH y las presentaciones atípicas que se pueden observar, constituye un reto hacer un diagnóstico ${ }^{(6)}$. Clínicamente los pacientes con coinfección leishmaniosis/SIDA tienen mayor tendencia a la afectación visceral, produciendo con cierta frecuencia lesiones cutáneas, que de otro modo son raras en la LV de la población inmuno competente ${ }^{(3,19,20)}$.

Estas lesiones cutáneas pueden ocurrir como consecuencia de la reactivación de una infección latente o como difusión de una infección reciente y pueden producir lesiones parecidas a la forma dérmica postkalazar $\left(\mathrm{PKDL}^{)(6,21)}\right.$.

En Europa los casos ocurridos en regiones de transmisión de L.infantum y que presentaron una variedad en las formas clínicas fueron asociados a pacientes VIH positivos con recuentos muy bajos de $\mathrm{CD} 4+{ }^{(6)}$, tal como se presenta el caso estudiado. En otro estudio realizado en Etiopía con 54 pacientes coinfectados Leishmania/VIH, ocho de ellos $(15 \%)$ presentaron lesiones atípicas: tres tenían lesiones cutáneas, dos lesiones orales, un paciente con lesión rectal y dos con afectación ganglionar ${ }^{(6)}$.

Es importante resaltar que estas presentaciones atípicas no están incluidas en las guías de diagnóstico y tratamiento de enfermedades oportunistas. Esto lleva consigo el riesgo por ejemplo que la LV atípica permanezca sin diagnosticar en el ámbito clínico, o se diagnostique con retraso ${ }^{(6)}$, esto disminuye la efectividad del tratamiento médico y aumentando las recidivas ${ }^{(20)}$.

La histoplasmosis es una de las micosis sistémicas más comunes en pacientes con SIDA es particular mente importante como coinfección oportunista donde es endémica. Las lesiones cutáneas son referidas como poco frecuentes en los pacientes diagnosticados en los Estados Unidos, se producen en menos del $10 \%$ de los casos. Por otro lado, entre los casos de América Latina, las lesiones de piel son reportadas en un $38 \%$ a $85 \%$ de los pacientes con histoplasmosis y $\operatorname{SIDA}^{(6,12,15,22)}$. Se cree que esto puede deberse a diferencias moleculares en cepas de H.capsulatum frecuentes en los Estados Unidos y América del Sur, lo que sugiere un tropismo del hongo a la piel en las cepas de Sudamérica ${ }^{(14,23)}$.

La histoplasmosis diseminada se producen los pacientes infectados por el VIH con un recuento de células $\mathrm{CD} 4+<50 / \mathrm{mL}$, tal como se encuentra en la mayoría de los estudios $^{(15)}$, así este paciente en el momento del diagnóstico presentaba un recuento de células CD4+de6/mL. Sin embargo, las lesiones cutáneas en histoplasmosis no son diagnósticas por sí mismas en pacientes inmuno deprimidos, como muchas otras enfermedades infecciosas y no infecciosas que comparten un patrón morfológico similar ${ }^{(15)}$.

Es importante el diagnóstico diferencial y a que en los pacientes con SIDA la histoplasmosis diseminada muestra signos y síntomas que se observan en otras enfermedades, como la tubérculo sismiliar, la leishmaniosis visceral, lacriptococosis diseminada, la paracoccidiomicosis y ciertos linfomas con comprometimiento intraabdominal, por otro lado en el caso de las lesiones muco-cutáneas pueden ser también similares a las que se presentan en la leishmaniosis tegumentaria, en la esporotricosis, sífilis, paracoccidiomicosis y tuberculosis ${ }^{(12)}$.

En el caso reportado, se ve la importancia de las lesiones de la piel como signo de una enfermedad sistémica y la inmuno supresión subyacente y esto también puede ayudar en el diagnóstico precoz de la histoplasmosis, permitir el tratamiento específico inmediato, y así reducir el riesgo de progresión de la enfermedad, que tiene una alta tasa de mortalidad en pacientes inmuno deprimidos ${ }^{(14)}$.

Con los hallazgos encontrados en este caso, concluimos que la presentación dérmica, en el paciente con diagnóstico previo de LV, fue causada por L. infantum, caracterizada por técnicas moleculares y por $H$. capsulatum que se presenta en concomitancia debido al estado inmunológico del paciente. Cabe resaltar la importancia del diagnóstico diferencial para aplicar el tratamiento correcto y además la relevancia epidemiológica ya que esta manifestación clínica normalmente no es asociada a esta especie de Leishmania. 


\section{REFERENCIAS BIBLIOGRÁFICAS}

1. Pérez A, Gala A, Rodríguez ME, Capó de Paz V, Collazo S, Fernández C. Histoplasmosis con manifestaciones cutáneas en pacientes Rev

Cubana MedTrop.2007;59(2):119-26.

2. Voss T, Mattos SL, Sementilli A, Romiti N, Beltrame PM, Veiga A. Atypical presentation of histoplasmosis in an immune compromised patient. An Bras Dermatol. 2015;90(1):32-5.

3. Pérez C, Solías $Y$, Rodríguez G. Leishmaniasis cutánea difusa en un paciente con sida. Biomédica 2006;26:485-97. Doi: http://dx.doi.org/10.7705/biomedica.v27i1. 241

4. Bañuls A, Hide M, Prugnolle F. Leishmania and the leishmaniases: a parasite genetic update and advances in taxonomy, epidemiology and pathogenicity in humans. AdvParasitol 2007;64:1-109.

5. Puig L, Pradinaud R. Leishmania and HIV coinfection: dermatological manifestations. Ann Trop Med Parasitol. 2003;97(1):10714.Doi: $10.1179 / 000349803225002589$

6. Diro E, Griensven J, Mohammed R, Colebunders $\mathrm{R}$, Asefa $\mathrm{M}$, et al. Atypical manifestations of visceral leishmaniasis in patients with HIV in north Ethiopia: a gap in guidelines for the management of opportunistic infections in resource poor settings. Lancet Infect Dis. 2015;15:1229.Doi: 10.1016/S1473-3099(14)70833-3

7. Cota GF, de Sousa MR, de Mendonça ALP, Patrocinio A, Assunção LS et al. LeishmaniaHIV Co-infection: Clinical Presentation and Outcomes in an Urban Area in Brazil. PLoS Negl Trop Dis. 2014; 8(4): e2816. Doi:http://dx.doi.org/10.1371/journal.pntd. 0002816

8. Rodgers MR, Popper SJ, Wirth DF. Amplification of kinetoplast DNA as a tool in the detection and diagnosis of Leishmania. Exp Parasitol. 1990;71(3):267-75.

9. Piarroux R, Gambarelli F, Dumon H, Fontes M, Dunan S, Mary C. et al. Comparison of PCR with direct examination of bone marrow aspiration, myeloculture, and serology for diagnosis of visceral Leishmaniasis in immune compromised patients. J Clin Microbiol. 1994;32(3):746-9.

10. Cáceres D, Gómez B, Restrepo A, Tobón A. Histoplasmosis y sida: factores de riesgo clínicos y de laboratorio asociados al pronóstico de la enfermedad. Infectio 2012;16(3):44-50.

Doi:http://dx.doi.org/10.1016/S01239392(12)70026-7

11. Frola C, Bermejo V, Spadaccini L, Guelfand $L$, Pérez H. Impacto de la histoplasmosis diseminada en pacientes HIV positivos. Actualizaciones en sida e infectología. 2013;21(80):37-41.
12. Ferreira MS, Borges AS. Histoplasmose. Rev. Soc. Bras. Med. Trop. 2009;42(2):192-8. http://dx.doi.org/10.1590/S003786822009000200020.

13. Vasudevan B, Ashish B, Amitabh S, A P M. Primary cutaneous histoplasmosis in a HIVpositive individual. J Glob Infect Dis. 2010 May;2(2):112-5. Doi: 10.4103/0974$777 X .62884$

14. Cunha VS, Zampese MS, Aquino VR, Cestari TF, Goldani LZ. Mucocutaneous manifestations of disseminated histoplasmosis in patients with acquired immunodeficiency syndrome: particular aspects in a Latin-American population. Clin Exp Dermatol 2007;32:250-5.

15. Marques S, Silvares M, Camargo R, Marques M. Cutaneous histoplasmosis disclosing an HIV-infection. An Bras Dermatol. 2013;88(3):420-3.

16. Orsi AT, Nogueira L, Chrusciak-Talhari A, Santos M, Ferreira LC, Talhari $S$ et al Histoplasmosis and AIDS co-infection. An Bras Dermatol. 2011;86:1025-6.

17. Saeki NM, SchubachAO, Salgueiro MM, Silva $F$, Wanke B, Lazera M. Histoplasmosecutânea primária: relato de caso em paciente imunocompetente e revisão de literatura. Rev. Soc. Bras. Med. Trop. 2008;41(6):680-2.Doi: http://dx.doi.org/10.1590/S003786822008000600024

18. Rodríguez G, Motta A, Ordóñez N. Estudio histopatológico de 19 biopsias cutáneas de pacientes con sida e histoplasmosis diseminada. Biomedica 2001;21:107-15.

19. Navarro M, Vilata J, Requena C, Aliaga A. Leishmaniasis mucocutánea en un paciente infectado con el VIH. Actas Dermosifiliogr. 2000;91:404-7.

20. Pascual I, Monasterio S, González-Obeso E, Corripio Sánchez R, Martínez Alegre J, PérezRobledo JP. Abdomen agudo en paciente VIH por Leishmaniasis visceral. Rev. Esp. Enferm. Dig. 2007;99(1):49-60.

21. Orsini M, Silva M, Profeta da Luz Z,Disch J, Fernades O, Moreira Det al. Identification of Leishmania chagasi from skin in Leishmania/HIV co-infection: a case report. Rev. Soc. Bras. Med. Trop. 2002;35(3):25962.

22. Kauffman CA. Histoplasmosis: a clinical and laboratory update. Clin Microbiol Rev. 2007;20(1):115-32.

23. Goldani LZ, Aquino VR, Lunardi LW, Cunha VS, Santos RP. Two specific strains of Histoplasmacapsulatum causing mucocutaneous manifestations of histoplasmosis: preliminary analysis of a frequent manifestation of histoplasmosis in southern Brazil. Mycopathologia. 2009;167(4):181-6. Doi: 10.1007/s11046008-9171-7. 